\title{
In silico structural analysis of group 3,6 and 9 allergens from Dermatophagoides farinae
}

\author{
FEIXIANG TENG, LILI YU, YONGHUA BIAN, JINXIA SUN, JUANSONG WU, \\ CUNBAO LING, LI YANG, YUNGANG WANG and YUBAO CUI
}

\begin{abstract}
Department of Laboratory Medicine, Yancheng Institute of Health Sciences, Yancheng, Jiangsu 224006, P.R. China
\end{abstract}
Received March 15, 2014; Accepted December 2, 2014

DOI: $10.3892 / \mathrm{mmr} .2015 .3166$

\begin{abstract}
Dermatophagoides farinae (Hughes; Acari: Pyroglyphidae) are the predominant source of dust mite allergens, which provoke allergic diseases, such as rhinitis, asthma and eczema. Of the 30 allergen groups produced by D. farinae, the Der f 3, Der f 6 and Der f 9 allergens are all trypsin-associated proteins, however little else is currently known about them. The present study used in silico tools to compare the amino acid sequences, and predict the secondary and tertiary structures of Der f 3, Der f 6 and Der f 9 allergens. Protein sequence alignment detected $\sim 46 \%$ identity between Der f 3, Der f 6 and Der f 9. Furthermore, each protein was shown to contain three active sites and two highly conserved trypsin functional domains. Predictions of the secondary and tertiary structure identified $\alpha$-helices, $\beta$-sheets and random coils. The active sites of the three proteins appeared to fold onto each other in a three-dimensional model, constituting the active site of the enzyme. Epitope analysis demonstrated that Der f 3, Der f 6 and Der f 9 have 4-5 potential epitopes located in random coils, and the epitope sequences of Der $\mathrm{f} 3$, Der f 6 and Der f 9 were shown to overlap in two domains (at amino acids 83-87 and 179-180); however the residues in these two domains were not identical. The present study aimed to conduct a biochemical and genetic analysis of these three allergens, and to potentially contribute to the development of vaccines for allergen-specific immunotherapy.
\end{abstract}

Correspondence to: Dr Yubao Cui, Department of Laboratory Medicine, Yancheng Institute of Health Sciences, 263 Jiefangnan Road, Yancheng, Jiangsu 224006, P.R. China

E-mail: ybcui1975@hotmail.com

Abbreviations: 3D, three-dimensional; IUIS, International Union of Immunological Societies; NCBI, National Center for Biotechnology Information; PDB, Protein Data Bank; IEDB, Immune Epitope Database and Analysis Resource

Key words: allergens, Dermatophagoides farinae, physiochemical characterization, secondary and tertiary structures, epitopes

\section{Introduction}

Dust mites are the predominant source of indoor allergens worldwide, and can exacerbate allergic diseases including asthma, rhinitis and dermatitis (1-3). Studies regarding the biological, chemical and structural properties of dust mite allergens have been critical for advancing the diagnosis and treatment of allergic disease $(4,5)$. In particular, allergens of the commonly found house dust mite Dermatophagoides farinae (Der f, Acari: Pyroglyphidae) have been extensively investigated. In crude extracts of $D$. farinae $>30$ components have been identified that can induce human immunoglobulin (Ig)E antibodies (4,6-7).

A total of 24 groups of dust mite allergens are currently listed in the International Union of Immunological Societies (IUIS) nomenclature dataset (http://www.allergen.org/). These allergens belong to various protein families, and three groups have been identified to possess trypsin activity: Group 3 comprises trypsins; group 6, chymotrypsins; and group 9, collagenolytic serine proteases (8). Previous studies have suggested that Der f 3, Der f 6 and Der f 9 are capable of activating the kallikrein-kinin system, which is involved in inflammation in normal human plasma (8-10). In addition, Der $\mathrm{f} 3$ has been shown to activate the complement system, resulting in the production of anaphylatoxins (11). Therefore, these allergen groups are likely important contributors to the hypersensitivity response produced by exposure to house dust mites.

Current research in immunotherapy for allergic disease is aimed at producing novel treatments that relieve the symptoms caused by hypersensitive responses (12). Allergen-specific immunotherapy (SIT) works by inducing the production of blocking antibodies, creating a shift toward a T-helper 1 response and inducing tolerance (13-14). SIT is able to induce IgG antibodies, which compete with IgE for allergen binding, thus inhibiting activation of IgE-dependent mast cells and basophils, and reducing IgE-mediated allergic inflammation (15-16). Conventional immunotherapies rely on crude dust mite extracts, however SIT is more successful with recombinant mite allergens (17). The use of recombinant allergens requires a good understanding of their structures and functions, in order to determine how they bind $\operatorname{IgE}$ and elicit an allergic response.

To improve understanding regarding the contributions of Der f 3, Der f 6, and Der f 9 to the allergenicity of D. farinae, the present study used a bioinformatics approach to compare 
amino acid sequences and predict their physiochemical characterizations, secondary structures, tertiary structures and B-cell epitopes. These analyses may aid the future development of SIT for Der f allergens.

\section{Materials and methods}

Sequence retrieval. The amino acid sequences of Der $\mathrm{f} 3$, Der $\mathrm{f} 6$ and Der $\mathrm{f} 9$ were retrieved from the International Union of Immunological Societies (IUIS) nomenclature database (18) and the National Center for Biotechnology Information protein sequence database (accession nos. BAA09920.1, AAF28423.1 and AAP35067.1, respectively). Signal peptide sequences were removed from the present analysis.

Physiochemical characterization. Physiochemical characteristics, including theoretical isoelectric point (pI), molecular weight, number of positively and negatively charged residues, extinction coefficients, instability index, aliphatic index and grand average of hydropathicity (GRAVY), were predicted using ExPASy ProtParam (19).

Primary and secondary structure analysis. DNAMAN version 6.0 software (Lynnon, Corp., Pointe-Claire, QC, Canada) was used to align the protein sequences. Prediction of transmembrane helices within the proteins was performed using the TMHMM 2.0 server (20). Predictions of the secondary structure ( $\alpha$-helices, $\beta$-sheets and random coils) were made using the Jpred 3 secondary structure prediction server (21). The active site and functional domains of the proteins were predicted using ExPASy PROSITE (19).

Three-dimensional (3D) model building and evaluation. Homology modeling and molecular dynamics were used to construct 3D structures of Der f 3, Der f 6 and Der f 9. Selecting an appropriate template is a critical stage to certify the quality of the final 3D structure. A Protein Basic Local Alignment Search Tool (BLASTP) online server (22) search, with default parameters, was performed against the Protein Data Bank (PDB), in order to identify suitable templates of Der $\mathrm{f} 3$, Der $\mathrm{f} 6$, and Der $\mathrm{f} 9$ for homology modeling. Following the BLASTP search, appropriate templates for generating 3D models of the proteins were selected, based on a high score, lower e-value and maximum sequence identity. BLAST results that indicated potential templates for modeling were PDB ID: 1A0J_A for Der $\mathrm{f} 3$, which displayed $32 \%$ amino acid sequence identity; and PDB ID: 1DST_A and PDB ID: 4D8N_A for Der $\mathrm{f} 6$ and Der $\mathrm{f} 9$ respectively, which displayed 32 and $34 \%$ amino acid sequence identities. Prediction of the 3D structure of Der f 3, Der $f 6$ and Der $\mathrm{f} 9$ was conducted using the homology-modeling program MODELLER version 9.12, as previously described $(23,24)$. Following alignment of the query and template proteins, and removal of potential errors, loop refinement was performed using the loop optimization method of MODELLER 9.12. The predicted structures were saved in PDB format, and energy minimization was performed using the GROMOS 96 force field in Swiss-PdbViewer, in order to rectify unfavorable clashes and improve the stereochemical quality (25).

Model evaluation is important to verify the quality of the 3D model. Therefore, PROCHECK (26) was used to evaluate the quality of the predicted models by Ramachandran plot assessment. Furthermore, model verification was performed using the overall quality factor (ERRAT) (27) and Verify 3D (28) programs. Superimposition of the query and template structures, and visualization of the generated 3D models was performed using UCSF Chimera 1.8 (29).

$B$-cell antibody epitope prediction. Amino acid sequences of Der f 3, Der $\mathrm{f} 6$ and Der $\mathrm{f} 9$ were submitted to Immune Epitope Database and Analysis Resource (IEDB) (30), ABCpred (31) and BepiPred (32) servers, in order to predict the possible linear B-cell epitopes with the default threshold. IEDB uses a collection of methods to predict linear B-cell epitopes, based on sequence characteristics of the antigen using amino acid scales and hidden Markov models (HMM). BepiPred predicts the location of linear B-cell epitopes, with a core epitope threshold set at 0.35 , using a combination of HMM and a propensity scale method. ABCpred predicts B-cell epitopes in an antigen sequence, and was the first server developed based on the recurrent neural network (machine-based technique), using fixed-length patterns with a default threshold of 0.51 . The predicted epitope regions were mapped to the predicted secondary and 3D protein structures, and DNAMAN 6.0 was used to generate a multiple-sequence alignment to identify the epitope regions of Der f 3, Der f 6 and Der f 9.

\section{Results}

Physiochemical characterization of Der $f 3, \operatorname{Der} f 6$ and Der $f$ 9. A comparison between the physiochemical characteristics of Der f 3, Der f 6 and Der f 9, as predicted using ProtParam, is presented in Table I. Der $\mathrm{f} 3$ and Der $\mathrm{f} 6$ were shown to possess similar characteristics; whereas fewer similarities were observed with Der $\mathrm{f} 9$. The $\mathrm{pI}$ values indicated that Der $\mathrm{f} 9$ is an alkaline protein, and Der $\mathrm{f} 3$ is more acidic, as compared with Der f 6. Extinction coefficients of Der f 3, Der f 6 and Der f 9 depended on the molar extinction coefficients of Tyr, Trp and Cys residues. Stability of Der f 3, Der f 6 and Der f 9 was investigated by analyzing the instability, aliphatic and GRAVY indices of these three proteins. The instability index value was lowest for Der $\mathrm{f} 6$, therefore Der f 6 is likely to be the most stable of the three allergens. The aliphatic index is defined as the relative volume occupied by aliphatic side chains (alanine, valine, isoleucine and leucine); and all three proteins were predicted to be fat-soluble. The GRAVY index indicated that all three proteins were also hydrophilic.

Protein sequence alignment and active site prediction. Protein sequence alignment was performed using the default parameters of DNAMAN 6.0. Der f 3, Der $\mathrm{f} 6$ and Der $\mathrm{f} 9$ exhibited a $45.61 \%$ sequence identity. Prosite prediction demonstrated that Der f 3 , Der $\mathrm{f} 6$ and Der $\mathrm{f} 9$ each contain an active site which is formed of a catalytic triad of histidine, aspartate and either serine or cysteine (Fig. 1). In addition, two sequence-specific trypsin functional domains of Der f 3, Der f 6 and Der f 9 (XTAAHC and XXCXGDS(C)GGPXV; bold indicates active site amino acid residues), which surround the histidine and serine/cysteine active sites, were shown to be highly conserved in the proteins (Fig. 1, domains I and II). 
Table I. Comparison of physiochemical characteristics of Der f 3, Der f 6 and Der f 9 allergens of Dermatophagoides farinae .

\begin{tabular}{lccc}
\hline Parameter & Derf 3 & Der f 6 & Der f 9 \\
\hline Accession no. & BAA09920.1 & AAF28423.1 & AAP35067.1 \\
Number of amino acids & 232 & 230 & 3334 \\
Total number of atoms & 3439 & 3491 & $\mathrm{C}_{1053} \mathrm{H}_{1672} \mathrm{~N}_{290} \mathrm{O}_{329} \mathrm{~S}_{9}$ \\
Formula & $\mathrm{C}_{1087} \mathrm{H}_{1710} \mathrm{~N}_{302} \mathrm{O}_{349} \mathrm{~S}_{10}$ & $\mathrm{C}_{1094} \mathrm{H}_{1738} \mathrm{~N}_{298} \mathrm{O}_{349} \mathrm{~S}_{12}$ & 23947.1 \\
Molecular weight (u) & 24913.9 & 25034.3 & 17 \\
Arg+Lys & 21 & 22 & 13 \\
Asp+Glu & 26 & 25 & 8.68 \\
Theoretical pI & 5.36 & $35785-35410$ & $35785-35410$ \\
Extinction coefficients & $38765-38390$ & & 32.69 \\
$\left(M^{-1}\right.$ cm ${ }^{-1}$ at $\left.260 \mathrm{~nm}\right)$ & & 29.24 & 88.84 \\
Instability index & 30.14 & 80.04 & -0.016 \\
Aliphatic index & 82.28 & -0.369 & \\
GRAVY & -0.284 & & \\
\hline
\end{tabular}

pI, isoelectric point; GRAVY, grand average of hydropathicity.

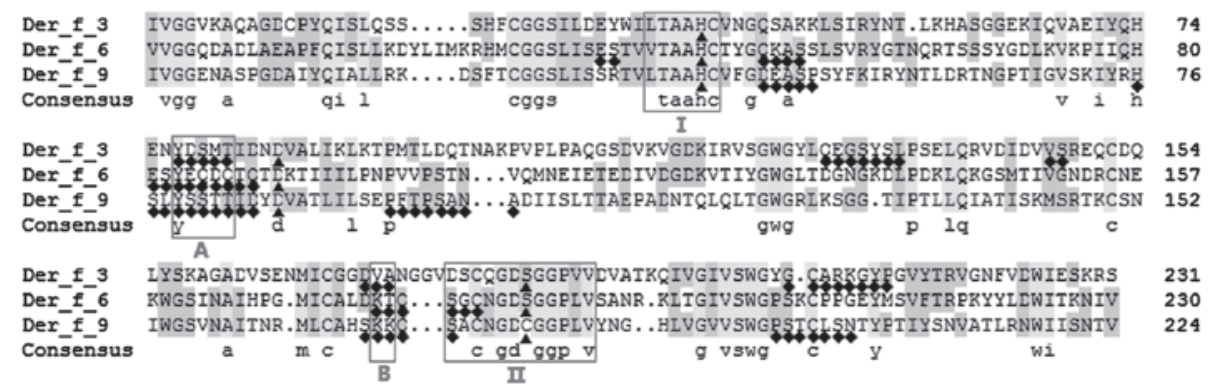

Figure 1. Amino acid sequence alignment of Der f 3, Der f 6 and Der $\mathrm{f} 9$ allergens of Dermatophagoides farinae. Active sites are indicated by ' $\mathbf{\wedge}$ ' and epitopes are indicated by ' $\checkmark$ '. The trypsin functional domains are contained in domains I and II, and overlapping epitopes are present in domains A and B.

Secondary structure prediction. The amino acid sequences of Der f 3, Der f 6 and Der f 9 were submitted to TMHMM 2.0, which is capable of predicting transmembrane helices. The three proteins were not predicted to contain any transmembrane helices, and localization was predicted to be outside of the membrane. Secondary structure prediction, using Jpred 3 , demonstrated that the three proteins contain $\alpha$-helices, $\beta$-sheets and random coils (Fig. 2). The largest number of residues was observed in the random coils, followed by the $\beta$-sheets and the $\alpha$-helices. More residues were detected in the $\alpha$-helices of Der f 3, as compared with Der f 6 and Der f 9. In addition, more residues were detected in the $\beta$-sheets of Der $f 6$, as compared with Der f 3 and Der f 9 (Table II).

$3 D$ model building, refinement, and evaluation. 3D structures of Der f 3, Der f 6 and Der f 9 were generated using MODELLER 9.12 with the following templates: PDB ID:1A0J_A, 1DST_A and 4D8N_A, respectively. The three models were viewed using Chimera 1.8, and a Ramachandran plot analysis of the models was conducted using PROCHECK. Analysis of the models indicated a high probability of confirmation, with $99.5,100$ and $100 \%$ of residues of Der f 3, Der f 6 and Der f 9, respectively, in the allowed region of the Ramachandran plot (Fig. 3). It is generally accepted that if $90 \%$ of residues are present in the allowed region of a Ramachandran plot, the model is reliable. Verification of the 3D analysis demonstrated that $94.85,84.42$ and $89.78 \%$ of the Der f 3, Der f 6 and Der f 9 residues, respectively, had an average $3 \mathrm{D}-1 \mathrm{D}$ score of $<0.2$, thus indicating that the three models were compatible with their sequences. Furthermore, the ERRAT of the models of Der f 3, Der f 6 and Der $f 9$ were $81.860,82.297$ and 84.722 , respectively. These results suggest that all computationally-generated models in the present study are considered to be of good quality and suitable for structural analysis.

The predicted 3D structures of Der f 3, Der f 6 and Der f 9 , like the secondary structures, were shown to contain $\alpha$-helices, $\beta$-sheets and random coils; however, the number of amino acids of these three structure elements differed slightly between the $3 \mathrm{D}$ and secondary structures. The percentage of overall amino acids located in $\alpha$-helices was 6.90 (two domains), 12.17 (four domains) and $10.27 \%$ (three domains) in Der f 3, Der f 6 and Der f 9, respectively; whereas the percentage of amino acids in $\beta$-sheets was 27.59 (13 domains), 26.96 (13 domains) and $30.80 \%$ (12 domains), respectively (Fig. 4). 3D structure overlap of the Der f 3, Der f 6 and Der f 9 proteins identified 
Table II. Secondary structure elements for Der f 3, Der f 6 and Der f 9 allergens of Dermatophagoides farinae.

\begin{tabular}{llll}
\hline Allergen & $\alpha$-helices $(\%)$ & $\beta$-sheets $(\%)$ & Random coils $(\%)$ \\
\hline Der f 3 & 5.60 (2 domains) & $29.74(13$ domains $)$ & 64.66 \\
Der f 6 & 5.22 (2 domains) & $32.17(14$ domains $)$ & 62.61 \\
Der f 9 & 4.91 (2 domains) & $30.36(14$ domains $)$ & 64.73 \\
\hline
\end{tabular}

\begin{tabular}{|c|c|}
\hline Der_f_3 & VGGVKAQAGDCPYQISLQSSSHECGGSILDEYWILTAAHCVNGQSAKKLSIRYNTLKHASGGEKIQVAEIYQHENYDSMT \\
\hline Der_f_3 & IDNDVALIKLKTPMTLDQTNA KPVPLPAQGSDVKVGDKIRVSGWGYLQEGSYSLPSELQRVDIDVVSREQCDQIYSKAGA \\
\hline Der_f_3 & GGVDSCQGDSGGPVVDVATKQIVGIVSWGYGCARKGYPGVYTRVGNFVDWIESKRSQ \\
\hline Der_f_6 & VGGQDADLAEAP FQISLLKDYIMKRHMCGGSLISESTVVTAAHCTYGQKASS LSVRYGTNRR: \\
\hline Der_f_6 & SYEQDQTQTDKTIIIILPNPVVPSTNVQMNEIETEDIVDGDKVTIYGWGLTDGNGKDLPDKLQKGSMTIVGNDRCNEKWGS \\
\hline Der_f_6 & INAIHPGMICALDKTQSGCNGDSGGPLVSANRKLTGIVSWGPSKCPPGEYMSVFTRPKYYLDWITKNIV \\
\hline Der_f_9 & VGGENASPGDAIYQIALLRKDS FTCGGSLISSRTVLTAAHCVFGDEASPSYFKIRYNTLDRTNGPITGVSKIYRHSLYSS \\
\hline Der_f_9 & TTIDYDVATLILSEPFTPSANADIISLTTAEPADNT DLLTGWGRLKSGGTIPTLL IATISKMSREKCSNIWGSVNAIT \\
\hline er & NRMLCAHSKKQSACNGDCGGPLVYNGHLVGVVSWGPSTCLSNTYPTIYSNVATLRNWIISNTV \\
\hline
\end{tabular}

Figure 2. Secondary structure elements of Der f 3, Der f 6, and Der f 9 allergens of Dermatophagoides farinae. $\alpha$-Helices are highlighted in black, $\beta$-sheets are circled, and unlabeled sequence represents random coils.

Table III. B-cell epitopes for Der f 3, Der f 6 and Der f 9 allergens of Dermatophagoides farinae.

\begin{tabular}{llc}
\hline Allergen & $\begin{array}{c}\text { Predicted } \\
\text { epitope sequences }\end{array}$ & $\begin{array}{c}\text { Peptide } \\
\text { numbers }\end{array}$ \\
\hline Derf 3 & YDSMT & $77-81$ \\
& QEGSYSL & $129-135$ \\
& VS & $147-148$ \\
Der f 6 & DVA & $172-174$ \\
& GCARKGYP & $206-213$ \\
& ES & $37-38$ \\
Der f 9 & QKAS & $50-53$ \\
& ESYEQDQTQ & $81-89$ \\
& KTQSGC & $175-180$ \\
& DEASP & $46-50$ \\
& HSLYSSTTID & $76-85$ \\
& PFTPSANA & $96-103$ \\
& SKKQS & $169-173$ \\
& PSTCLSN & $197-203$ \\
\hline
\end{tabular}

more overlapping in the $\alpha$-helix and $\beta$-sheet domains, as compared with the random coil domains. The three active sites of Der f 3, Der f 6 and Der f 9 were shown to completely overlap within the $3 \mathrm{D}$ structure, and fold onto each other to constitute the active site of the enzyme (Fig. 4).

The predicted secondary and 3D structures of Der $\mathrm{f} 3$, Der $f 6$ and Der 99 had slightly different compositions. Peptides $103-105$ coiled into a $\beta$-sheet in the predicted secondary structure of Der f 3; however, this was not observed in the 3D structure prediction. In Der f 6, peptides 109-110 coiled into $\beta$-sheets only in the predicted secondary structure; whereas peptides 45-49 and 181-183 coiled into $\alpha$-helices and 187-190 into a $\beta$-sheet only in the predicted 3D structure. In Der f 9 , peptides $104-106$ coiled into a $\beta$-sheet only in the secondary structure; whereas peptides $40-43$ coiled into an $\alpha$-helix and 164-168 into a $\beta$-sheet only in its 3D structure.

Predicted B-cell epitopes. B-cell epitopes of Der f 3, Der f 6, and Der f 9 were predicted using IEDB Analysis Resource, ABCpred and BepiPred, and were verified in the predicted secondary and 3D structures. Der f 3, Der f 6 and Der f 9 were predicted to contain 5, 4 and 5 main potential epitopes, respectively, all located in random coils (Table III). Amino acid sequence alignment and overlapping 3D structures demonstrated an overlap in the epitopes of Der $\mathrm{f} 3$, Der $\mathrm{f} 6$ and Der f 9 (Fig. 1; domains A and B, peptides 83-87 and 179-180; Fig. 4D), however the residues in two overlapping epitope sequences were not identical.

\section{Discussion}

Allergic diseases are one of the most common types of human disease. Besides avoiding allergen exposure, allergen-specific immunotherapy (SIT) is the only curative treatment option (33). Patients respond to symptomatic pharmacotherapy, such as antihistamines and corticosteroids, however the majority of patients report inadequate symptom relief (34). SIT provides an alternative disease-specific treatment, which induces tolerance to the allergen (35). SIT can result in long-term remission of allergic symptoms and may reduce the chances of developing novel sensitization to other allergens (17).

Recently, the increased prevalence of allergic diseases has placed greater demand on diagnostic and therapeutic products; however, crude mite allergen extracts that are applied for diagnostic and therapeutic purposes do not meet the demand (36). Crude extracts include non-allergenic antigens and ineffective concentrations of important allergens, resulting in numerous side effects (37). The use of recombinant allergens offers a 

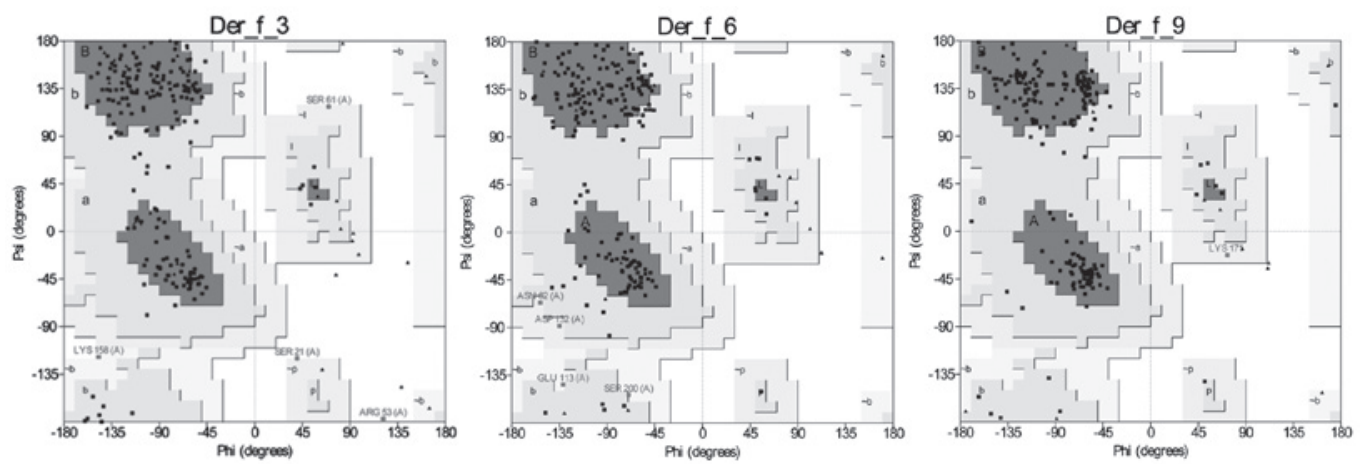

Figure 3. Ramachandran plot analysis of Der f 3, Der f 6, and Der f 9 allergens of Dermatophagoides farinae.

A

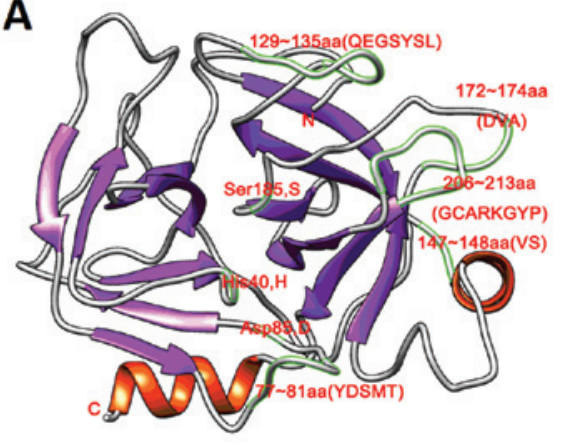

C

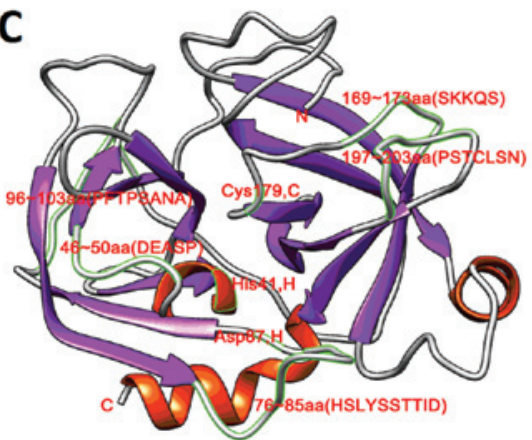

B

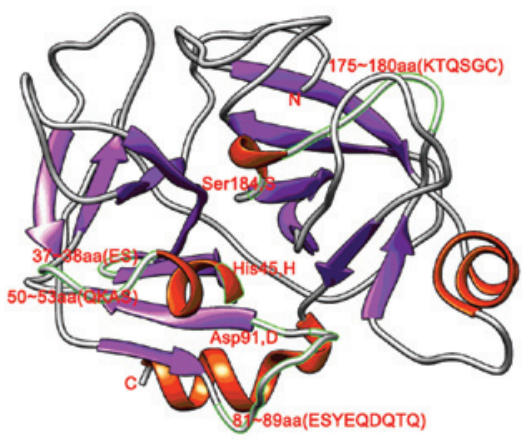

D

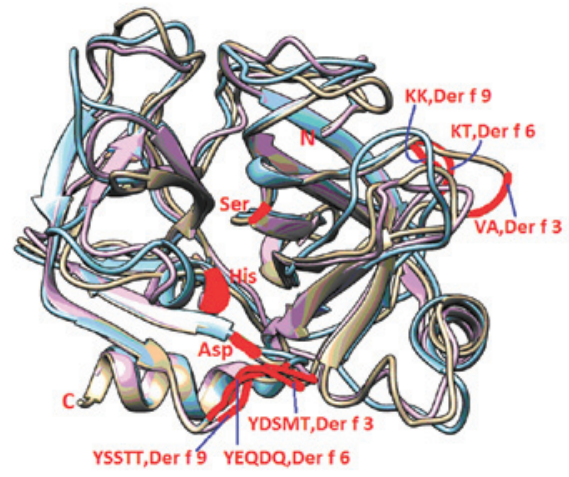

Figure 4. Homology-modeled structures of Der f 3, Der f 6, and Der f 9 allergens of Dermatophagoides farinae. (A) Secondary structure of Der f 3 contains two $\alpha$-helices and $13 \beta$-sheets. Histidine, aspartate and serine formed into catalytic triad of active sites in Der f 3 , which fold onto each other in the $3 \mathrm{D}$ model. (B) Secondary structure of Der f 6 contains four $\alpha$-helices and $13 \beta$-sheets. Histidine, aspartate and serine formed into catalytic triad of active sites in Der f 6, which fold onto each other in the 3D model. (C) Secondary structure of Der f 9 contains three $\alpha$-helices and $12 \beta$-sheets. Histidine, aspartate and cysteine formed into catalytic triad of active sites in Der f 9 , which fold onto each other in the 3D model. $\alpha$-helices are shown in orange, $\beta$-sheets in purple and random coils in gray. (D) 3D structural overlap of Der f 3, Der f 6, and Der f 9 revealed more overlapping in $\alpha$-helix and $\beta$-sheet domains, whereas there was less overlapping in $\beta$-turn and random coil domains. Active site residues of Der f 3 , Der f 6 , and Der f 9 overlapped completely in the 3D structure and folded onto each other to constitute catalytic triad of active sites. Der $\mathrm{f} 3$ is shown in light brown, Der $\mathrm{f} 6$ in light blue and Der f 9 in purple. The active sites and overlapping epitopes are shown in red.

better prospect for the rational and accurate diagnosis and treatment of allergies. Pure and standardized recombinant allergens contain the majority of the IgE-binding epitopes of an allergen source, and can be formulated to replace natural extracts (38). The use of recombinant allergens in diagnosis allows the exact identification of the molecules that are provoking the allergic reaction (37). Der f 3, Der f 6 and Der f 9 allergens are currently listed in the IUIS nomenclature dataset, and developing recombinant allergens of these three proteins may contribute to the diagnosis and treatment of mite-induced allergic diseases.

The results of the present study indicated that Der $\mathrm{f} 9$ is alkaline, whereas Der f 3 and Der f 6 are acidic. Der f 3 and
Der f 6 were shown to possess more similar characteristics to one another, as compared with Der f 9. These three proteins are $45.61 \%$ identical in amino acid sequence and contain three active sites: His, Asp and Ser/Cys, which are common residues constituting the active site of a protease. A 3D analysis indicated that these sites are likely to fold onto each other, constituting the active site of the enzyme. Furthermore, two trypsin functional domains were identified in Der $\mathrm{f} 3$, Der $\mathrm{f} 6$ and Der f 9 (XTAAHC and XXCXGDS(C)GGPXV), which have high amino acid sequence identity and were shown to surround the His and Ser/Cys active sites.

Secondary and tertiary protein structures are important for the prediction of epitopes. $\alpha$-Helices and $\beta$-sheets are two 
common secondary structures of protein, whose conformations are maintained by hydrogen bonds, rendering it unlikely for epitope sequences to be located within them. Conversely, random coils are located in the surface-exposed region proteins, and often contain epitope sequences (39). In the present study, the secondary and tertiary structures of Der $\mathrm{f} 3$, Der f 6 and Der f 9 were predicted to contain $\alpha$-helices, $\beta$-sheets and random coils. Epitope prediction identified 4-5 potential epitopes located in the random coils of each of the three allergens. Furthermore, the epitope sequences of Der $\mathrm{f} 3$, Der $\mathrm{f} 6$ and Der $\mathrm{f} 9$ were shown to overlap in two domains. However, the amino acids in these two domains were not $100 \%$ identical, this is likely a reflection of the $<50 \%$ identity between Der f 3, Der f 6 and Der f 9 sequences.

In conclusion, the present study conducted a biochemical and genetic analysis of the Der f 3, Der f 6 and Der f 9 allergens. The results may provide a basis for further studies of the allergenicity and function of these three allergens, and may contribute to the development of a vaccine for allergen-specific immunotherapy.

\section{Acknowledgements}

The present study was supported by the National Sciences Foundation of China (grant nos. NSFC31272369 and NSFC81001330) and the Jiangsu Provincial Health Department (grant no. Z200914).

\section{References}

1. Milián E and Díaz AM: Allergy to house dust mites and asthma. P R Health Sci J 23: 47-57, 2004.

2. Nadchatram M: House dust mites, our intimate associates. Trop Biomed 22: 23-37, 2005.

3. Bunnag C, Jareoncharsri P, Tantilipikorn P, Vichyanond P and Pawankar R: Epidemiology and current status of allergic rhinitis and asthma in Thailand - ARIA Asia-Pacific Workshop report. Asian Pac J Allergy Immunol 27: 79-86, 2009.

4. Cui Y: Structural biology of mite allergens. Mol Biol Rep 40: 681-686, 2013

5. Morales M, Iraola V, Leonor JR and Carnés J: Enzymatic activity of allergenic house dust and storage mite extracts. J Med Entomol 50: 147-54, 2013.

6. Thomas WR, Smith WA, Hales BJ, Mills KL and O'Brien RM: Characterization and immunobiology of house dust mite allergens. Int Arch Allergy Immunol 129: 1-18, 2002.

7. Thomas WR, Smith WA and Hales BJ: The allergenic specificities of the house dust mite. Chang Gung Med J 27: 563-569, 2004.

8. Cui Y, Zhou Y, Shi W, et al: Molecular cloning, expression, sequence analyses of dust mite allergen Der $\mathrm{f} 6$ and its IgE-binding reactivity with mite allergic asthma patients in southeast China. Mol Biol Rep 39: 961-968, 2012.

9. Takahashi K, Aoki T, Kohmoto S, et al: Activation of kallikrein-kinin system in human plasma with purified serine protease from Dermatophagoides farinae. Int Arch Allergy Appl Immunol 91: 80-85, 1990.

10. Polosa R: Role of the kinin-kallikrein pathway in allergic diseases. Allergy 48: 217-225, 1993.

11. Maruo K, Akaike T, Ono T, Okamoto T and Maeda H: Generation of anaphylatoxins through proteolytic processing of $\mathrm{C} 3$ and C5 by house dust mite protease. J Allergy Clin Immunol 100: 253-260, 1997
12. Schei MA, Hessen JO and Lund E: House-dust mites and mattresses. Allergy 57: 538-542, 2002.

13. Durham SR and Till SJ: Immunologic changes associated with allergen immunotherapy. J Allergy Clin Immunol 102: 157-164, 1998.

14. Valenta R: The future of antigen-specific immunotherapy of allergy. Nat Rev Immunol 2: 446-453, 2002.

15. Larché M, Akdis CA and Valenta R: Immunological mechanisms of allergen-specific immunotherapy. Nat Rev Immunol 6: 761-771, 2006.

16. Kowalski ML and Jutel M: Mechanisms of specific immunotherapy of allergic diseases. Allergy 53: 485-492, 1998.

17. Cappella A and Durham SR: Allergen immunotherapy for allergic respiratory diseases. Hum Vaccin Immunother 8: 1499-1512, 2012.

18. Larsen JN and Løwenstein H: Allergen nomenclature. J Allergy Clin Immunol 97: 577-578, 1996.

19. Wilkins MR, Gasteiger E, Bairoch A, et al: Protein identification and analysis tools in the ExPASy server. Methods Mol Biol 112: 531-552, 1999.

20. Krogh A, Larsson B, von Heijne G, and Sonnhammer EL: Predicting transmembrane protein topology with a hidden Markov model: application to complete genomes. J Mol Biol 305: 567-580, 2001.

21. Cole C, Barber JD and Barton GJ: The Jpred 3 secondary structure prediction server. Nucleic Acids Res 36: W197-W201, 2008.

22. Altschul SF, Madden TL, Schäffer AA, et al: Gapped BLAST and PSI-BLAST: a new generation of protein database search programs. Nucleic Acids Res 25: 3389-3402, 1997.

23. Sali A and Blundell TL: Comparative protein modeling by satisfaction of spatial restraints. J Mol Biol 234: 779-815, 1993.

24. Eswar N, Webb B, Marti-Renom MA, et al: Comparative protein structure modeling using Modeller. Curr Protoc Bioinformatics Chapter 5: Unit 5.6. doi: 10.1002/0471250953. bi0506s15, 2006

25. Guex N and Peitsch MC: SWISS-MODEL and the Swiss-PdbViewer: an environment for comparative protein modeling. Electrophoresis 18: 2714-2723, 1997.

26. Laskowski RA, MacArthur MW, Moss DS and Thornton JM: PROCHECK: A program to check the stereochemical quality of protein structures. J Appl Cryst 26: 283-291, 1993.

27. Colovos C and Yeates TO: Verification of protein structures: patterns of non-bonded atomic interactions. Protein Sci 2: 1511-1519, 1993.

28. Lüthy R, Bowie JU and Eisenberg D: Assessment of protein models with three-dimensional profiles. Nature 356: 83-85, 1992.

29. Pettersen EF, Goddard TD, Huang CC, et al: UCSF Chimera - a visualization system for exploratory research and analysis. J Comput Chem 25: 1605-1612, 2004.

30. Vita R, Peters B and Sette A: The curation guidelines of the immune epitope database and analysis resource. Cytometry A 73: 1066-1070, 2008.

31. Saha S and Raghava GP: Prediction of continuous B-cell epitopes in an antigen using recurrent neural network. Proteins 65: 40-48, 2006.

32. Larsen JE, Lund $\mathrm{O}$ and Nielsen M: Improved method for predicting linear B-cell epitopes. Immunome Res 2: 2, 2006.

33. Klimek L and Pfaar O: A comparison of immunotherapy delivery methods for allergen immunotherapy. Expert Rev Clin Immunol 9: 465-474, 2013.

34. Wu AY: Immunotherapy - Vaccines for allergic diseases. J Thorac Dis 4: 198-202, 2012.

35. Frew AJ: Allergen immunotherapy. J Allergy Clin Immunol 125: S306-S313, 2010.

36. Cui Y,Zhou Y,Ma G, et al: Cloning, bioinformatics analysis, and expression of the dust mite allergen Der 5 of Dermatophagoides farinae. Braz J Med Biol Res 45: 746-752, 2012.

37. Schmidt G, Gadermaier G, Pertl H, et al: Production of recombinant allergens in plants. Phytochem Rev 7: 539-552, 2008.

38. Larché M: Peptide and recombinant immunotherapy. Immunol Allergy Clin North Am, 31: 377-389, 2011.

39. Sikic K, Tomic S and Carugo O: Systematic comparison of crystal and NMR protein structures deposited in the protein data bank. Open Biochem J 4: 83-95, 2010. 